\title{
甘油作绿色溶剂在有机合成中的应用研究进展
}

\author{
张霞忠 ${ }^{a}$ 邹润英 ${ }^{a}$ 埅家英 ${ }^{a}$ 周文俊 ${ }^{*}, a$ 王进贤 $b$ \\ ( ${ }^{a}$ 内江师范学院化学化工学院 内江 641112) \\ ( ${ }^{b}$ 西北师范大学化学化工学院 兰州 730070)
}

\begin{abstract}
摘要 大多数有机反应都是在溶剂中进行的, 反应溶剂的使用不仅有利于反应物和催化剂的接触, 也决定工作过程和 处理策略的选择. 甘油作为生物柴油工业的主要副产物，具有低毒、高沸点、非可燃、非腐蚀、难挥发、化学性质稳 定的特性，这些性质符合理想绿色溶剂的大部分要求. 同时，甘油作为溶剂使用，既有原料保障又没有消耗石油资源， 完全符合当前发展可持续化学过程的要求. 近年来, 以甘油作为绿色新型溶剂, 实现了很多有机反应, 包括加成、还 原、偶联、多组分反应等. 在部分的有机反应中, 使用甘油作为绿色溶剂, 不仅能够提高反应的产率或改变反应的选择 性, 同时还可以实现溶剂的回收循环使用. 本文主要介绍近年来甘油作为绿色溶剂在加成反应、还原反应、偶联反应 和多组分反应等有机反应领域中的应用. 通过这个视角论述这些应用实例, 期望能为甘油在有机合成中更好的应用提 供启发.
\end{abstract}

关键词 生物柴油; 甘油; 绿色溶剂; 有机合成

\section{Recent Progress of Glycerol as Green Solvents in Organic Synthesis}

\author{
Zhang, Xiazhong $^{a} \quad$ Zou, Runying $^{a} \quad$ Deng, Jiaying $^{a} \quad$ Zhou, Wenjun $^{*, a} \quad$ Wang, Jinxian ${ }^{b}$ \\ ( ${ }^{a}$ College of Chemistry and Chemical Engineering, Neijiang Normal University, Neijiang 641112) \\ ( ${ }^{b}$ College of Chemistry and Chemical Engineering, Northwest Normal University, Lanzhou 730070)
}

\begin{abstract}
Traditionally, organic synthesis is carried out in solvents, which not only facilitate the intimate contact of reactants and catalysts but also determines the choice of work up procedures and disposal strategies. Glycerol (1,2,3-propanetriol or glycerin) is easily available as the main by-product of the biodiesel industry. The increased production in the synthesis of biofuel, i.e., biodiesel, has led to a growth in the supply of glycerol, which is a non-toxic and recyclable liquid that is highly inert and stable, and compatible with many other non-irritating and non-toxic chemicals. According to the twelve principles of green chemistry, the features of glycerol are to meet the criteria for green solvents. Recently, glycerol has attracted attention as it is a versatile, cheap, and renewable feedstock and has been proven to be an environmentally friendly reaction media for the synthetic organic chemistry. In many reactions, glycerol as green solvent can improve the yield of the reaction or change the selectivity of the reaction. In order to provide some inspiration of better organic synthetic method design for glycerol, the use of glycerol as a green solvent in organic synthesis is reviewed, such as addition reactions, reduction reactions, coupling reactions, multi-component reactions, and so on.
\end{abstract}

Keywords biodiesel; glycerol; green solvent; organic synthesis

有机反应大多数都在液相中发生, 反应溶剂是重要 组成成分并且其使用量较大. 在有机合成化学中, 溶剂 能促进反应物和催化剂的紧密接触，同时还决定了反应 的历程以及溶剂的回收. 考虑到化学反应对环境的影 响, 寻找替代挥发性有机溶剂的绿色溶剂成为学术界和 工业界的巨大挑战 ${ }^{[1]}$.
根据绿色化学的十二项原则, 一种理想的绿色溶剂 应具有高沸点、低蒸气压、低毒等性质，还应有价廉、 不易燃、可回收再利用等优点 ${ }^{[2]}$, 但满足所有条件的绿 色溶剂并不存在. 在过去的十年中, 已找到了一些替代 溶剂(水、离子液体、超临界流体: 超临界二氧化碳和全 氟化溶剂等 $)^{[3]}$. 但由于各种原因, 还没有出现一种普遍

*E-mail: zhwj84@126.com, zhouwj@njtc.edu.cn

Received October 30, 2014; revised December 15, 2014; published online January 15, 2015.

Project supported by the National Natural Science Foundation of China (No. 20572086), the Sichuan Province Education Office (Nos. 12ZB074, 13ZB0002), and the Startup Foundation of Ph. D. Scientific Research of Neijiang Normal University.

国家自然科学基金(No. 20572086)、四川省教育厅(Nos. 12ZB074, 13ZB0002)和内江师范学院博士科研启动费(No. 2012B05)资助项目. 
的绿色溶剂, 科研界正不断地寻找广泛适用于催化反 应、有机反应的新溶剂.

随着可持续发展的观念深入人心, 石油和煤等化石 资源的日益枯竭以及使用过程中造成的环境污染问题, 已成为制约社会发展的瓶颈. 这些资源和环境问题促使 人们研究可再生的生物质资源. 伴随着生物柴油产业的 快速发展, 主要副产品甘油的产量不断增长 ${ }^{[4]}$. 生物柴 油的生成是一个相对简单的过程, 碱性条件下, 甘油三 酯和短链的脂肪族醇(通常是甲醇)发生催化酯交换反应 生成甘油和三种酯(Eq. 1) ${ }^{[5]}$. 甘油是一种味甜、无色、无 嗅的粘性液体, 物理性质独特, 具有高沸点、低蒸气压、 高介电常数、不易燃、强极性、可生物降解, 能与水、 短链醇混溶, 微溶于许多常见的有机溶剂(乙酸乙酯、二 氯甲烷、乙醚等)而不溶于烃类等性质, 其突出的优点是 能与不溶于水的有机化合物互溶 ${ }^{[6]}$. 它低毒、非腐蚀、 非可燃、难挥发、宽液程等特征符合理想绿色溶剂的大 部分要求.<smiles>[R]C(=O)OCC(COC([R])[R])OC(=O)OC</smiles>
$R=R^{1}, R^{2}, R^{3}=$ hydrocarbon chain from 15 to 21 carbon atoms

一直以来, 人们非常重视对甘油的开发和探索. 其 主要的研究工作是将甘油转换成有附加值的化学制品 和甘油重整制氢的发展过程. 近年来, 甘油作为绿色反 应介质, 引起了化学界的广泛关注. 自 Wolfsons 等 ${ }^{[7]}$ 发 现甘油可以作为绿色反应溶剂在有机合成中使用以后, 甘油作为溶剂在有机反应中的应用越来越多 ${ }^{[8]}$. 本文对 近年来甘油作为绿色溶剂的应用进行总结, 主要介绍其 在加成反应、还原反应、偶联反应和多组分反应等有机 反应领域中的应用.

\section{2 加成反应}

\subsection{Michael 加成反应}

2008 年, $\mathrm{Gu}$ 等 ${ }^{[9]}$ 研究了甘油中对甲氧基苯胺与 $\alpha, \beta$ 不饱和羰基化合物的 $a z a$-Michael 加成反应. 该反应在 无催化剂条件下, $100{ }^{\circ} \mathrm{C}$ 反应 $20 \mathrm{~h}$ 即可以较高产率 (82\%)得到目标产物. 同时, 他们还发现腈类化合物也 能在该条件下发生 $a z a$-Michael 加成反应(Scheme 1). 许 多有机溶剂[如甲苯、 $N, N$-二甲基甲酰胺(DMF)、二甲亚 砜(DMSO)等]对上述反应不起作用. 以水为溶剂也仅有 少量产物生成. 此外, 甘油经回收重复使用 3 次后仍能 获得较好的收率.

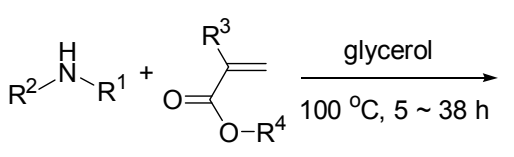

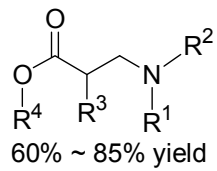

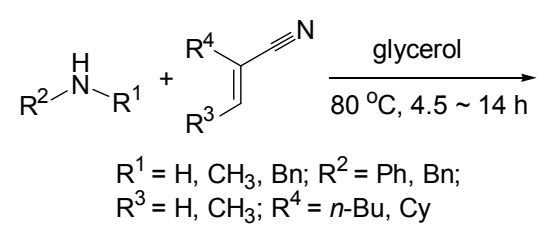<smiles>[R][R]</smiles>

$72 \% \sim 93 \%$ yield $\mathrm{R}^{3}=\mathrm{H}, \mathrm{CH}_{3} ; \mathrm{R}^{4}=n-\mathrm{Bu}, \mathrm{Cy}$

图式 1 胺与 $\alpha, \beta$-不饱和羰基化合物或腈类化合物的 $a z a-$ Michael 加成反应

Scheme 1 The $a z a$-Michael addition reaction of amines to $\alpha, \beta$ unsaturated carbonyl compound or nitrile compound

吲哚衍生物与硝基苯乙烯的 Michael 加成反应也能 在甘油中得以进行, 且能获得较高产率的产物(Eq. 2$)^{[9]}$. 值得一提的是，该反应无需任何催化剂即可进行，而使 用其他有机溶剂(如甲苯、DMF)代替甘油只能得到很少 的产物.

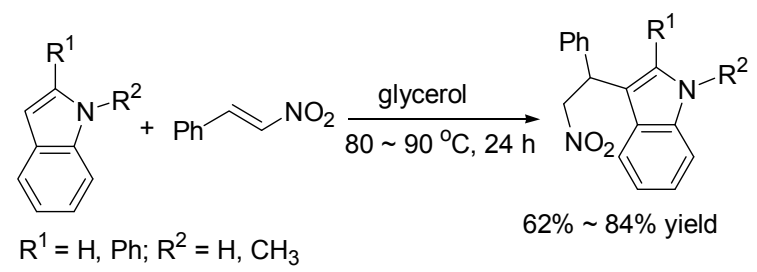

苯硫酚和柠檬醛可以在甘油中发生加成反应 (Scheme 2), 并且, 通过催化剂的选择可以成功实现反 应的调控 ${ }^{[10]}$. 以 $\mathrm{KF} / \mathrm{Al}_{2} \mathrm{O}_{3}$ 为催化剂, 反应有利于 1,4 -加 成物的形成, 而采用 $\mathrm{H}_{2} \mathrm{SO}_{4}$ 为催化剂时, 主要得到 1,2加成产物. 其中 $\mathrm{KF} / \mathrm{Al}_{2} \mathrm{O}_{3}$ 作催化剂时, 反应的适用范围 很广, 脂肪族硫醇、芳香族硫醇都能与柠檬醛发生 1,4加成.

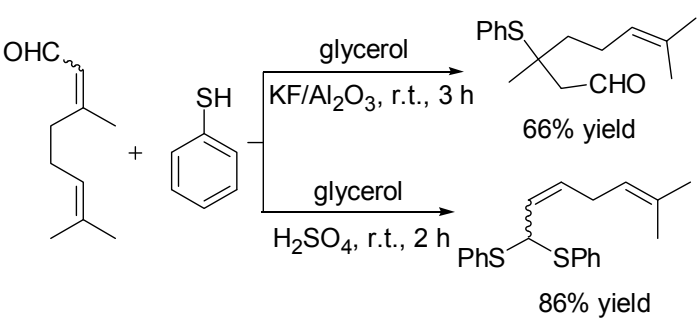

图式 2 苯硫酚和柠檬醛的 Michael 加成反应

Scheme 2 The Michael addition reaction of thiophenol to citral

\section{2 羟醛缩合反应}

Wolfson 等 ${ }^{[11]}$ 成功地完成了甘油中的羟醛缩合反应 (Eq. 3). 在 $80{ }^{\circ} \mathrm{C}$ 条件下, 以 $\mathrm{KOH}$ 为催化剂, 正戊醛能 发生自身的羟醛缩合反应，其产率可达 $52 \%$. 


$$
\underset{(2 \text { equiv. })}{\frac{\text { glycerol, } \mathrm{KOH}}{80^{\circ} \mathrm{C}, 2 \mathrm{~h}}}
$$

\section{3 开环反应}

在用环氧乙烷催化水合法制备乙二醇工艺中, 甘油 可以作为反应的有效溶剂(Eq. 4) ${ }^{[12]}$, 研究发现甘油的加 入可以有效提升反应的转化率和选择性. 在 $\mathrm{N}_{2}$ 充压 1.0 $\mathrm{MPa}, \mathrm{NY} 3$ 催化剂 $20 \mathrm{~g}$ (不计溶剂时, 质量百分含量为 $6.25 \%$ ), 水比 $\geqslant 4.6$, 甘油量在 $20 \sim 45 \mathrm{~g}$ 之间, 反应温度 控制在 $120 \sim 140^{\circ} \mathrm{C}$, 反应时间约 $20 \mathrm{~min}$, 产品的转化率 在 $99 \%$ 以上，选择性高达 $95 \%$.

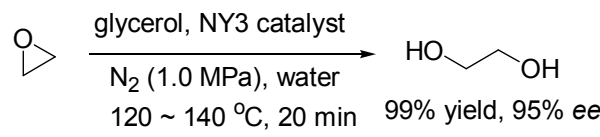

苯基环氧乙烷与对甲氧基苯胺的开环反应在水中 或甘油中都能顺利发生(Eq. 5), 但是该反应选择性受溶 剂影响比较大 ${ }^{[9]}$. 研究表明甘油为溶剂时, 该反应的选 择性明显高于以水为溶剂时的选择性.

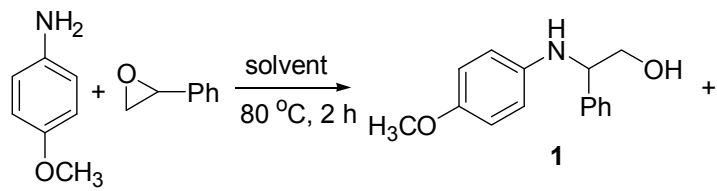<smiles>COc1ccc(NCC(O)c2ccccc2)cc1</smiles>

In glycerol: $85 \%$ yield, ratio $\mathbf{1 / 2}=93 / 7$ In water: $88 \%$ yield, ratio $1 / 2=76 / 24$

Jérôme 等 ${ }^{[13]}$ 研究了以天然高分子氨基多糖 3 (AP) (图 1)作催化剂, 在甘油中实现了环氧化合物与脂肪酸 的开环反应, $3 \mathrm{~h}$ 内, 以 $85 \% \sim 98 \%$ 产率得到相应的酯 (Eq. 6). 该催化剂循环使用十次以上, 未发现其活性降 低. 传统催化剂, 如 $\mathrm{ZnO}, \mathrm{K}_{2} \mathrm{CO}_{3}$ 等, 反应时间长, 产率 低, 选择性差. 进一步研究表明, 用附在甘油表面的树 状聚丙烯亚胺 4 (PPI)作为催化剂(图 2), 也能发生选择 性开环反应生成相应的酯 ${ }^{[14]}$.

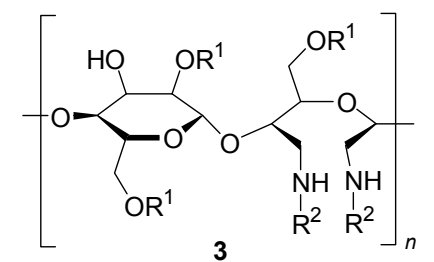

$\mathrm{R}^{1}=\left(\mathrm{CH}_{2} \mathrm{CH}_{2} \mathrm{O}\right)_{n} \mathrm{H} ; \mathrm{R}^{2}=\left(\mathrm{CH}_{2}\right)_{11} \mathrm{CH}_{3}$

图 1 氨基多糖(AP)的结构式

Figure 1 The structure of aminopolysaccharide (AP)

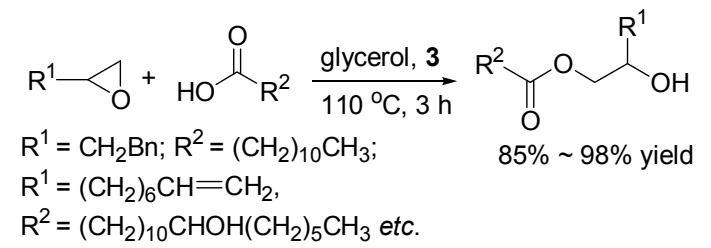<smiles>[R]N([R])CCCCN([R])[R]</smiles>

$\mathrm{R}^{1}=\left(\mathrm{CH}_{2}\right)_{3} \mathrm{~N}\left(\mathrm{CH}_{2} \mathrm{CH}_{2} \mathrm{CH}_{2} \mathrm{NHR}^{2}\right)_{2}$<smiles>[R]C[C@H](C)C(=O)OCC(O)CO</smiles>

图 2 聚丙烯亚胺(PPI)的结构式

Figure 2 The structure of polypropylene imine (PPI)

\section{4 缩合反应}

以甘油为溶剂, 无催化剂条件下, 2-甲基吲哚与醛 类化合物作用能有效地合成双吲哚甲烷衍生物 (Eq. $7)^{[15]}$. 若该反应使用甲苯、正丁醇、DMF、DMSO 等作 溶剂，在无催化剂的条件下其产率很低, $\mathrm{CeCl}_{3}$ 的加入能 有效促进反应的进行 ${ }^{[16]}$. 此外, 该反应适用范围很广, 芳香醛、杂环醛和脂肪醛等均可顺利完成反应. 由于反 应产物不溶于甘油，反应结束后通过简单的过滤即可实 现分离.

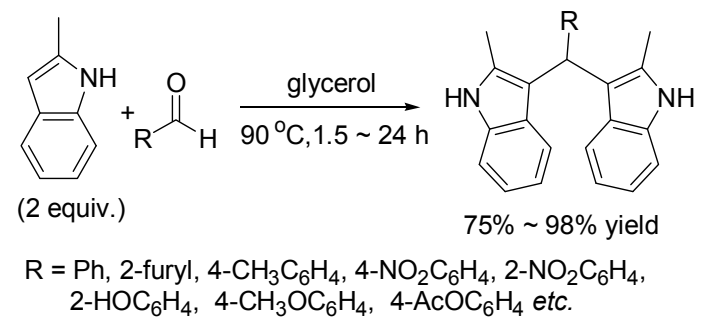

甘油中，对乙酰氨基苯甲醛与 1,3-环己二酮可以快 速地发生缩合，以较高的产率得到相应的氧杂蒽衍生物 $(85 \%)(\text { Eq. } 8)^{[15]}$. 若该反应在甲苯、DMF、DMSO、 $\mathrm{CH}_{3} \mathrm{NO}_{2}$ 等有机溶剂中进行, 只会得到微量的产物 $(<5 \%)$. 甘油在该反应中显示出如此独特的效果, 致使 人们开始猜测是不是由于甘油中三个羟基形成的氢键 网络在起作用.
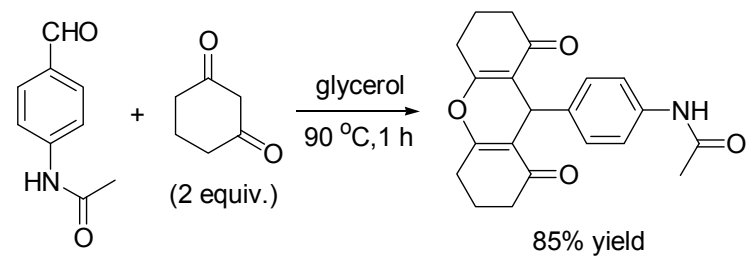

同样，以甘油作为溶剂，无催化剂条件下，通过芳 
醛、杂环醛或 $\alpha, \beta$-不饱和醛与 1,3-环己二酮的缩合反应 可以制备一系列氧杂葱衍生物. 反应物如果是水杨醛, 该反应通常认为要在酸性催化剂存在的条件下才能进 行, 但是选用甘油作溶剂, 在无催化剂条件下, 通过延 长反应时间 $(6 \sim 12.5 \mathrm{~h})$ 即可以较高的收率得到相应的目 标产物(Eq. 9 $)^{[15]}$.

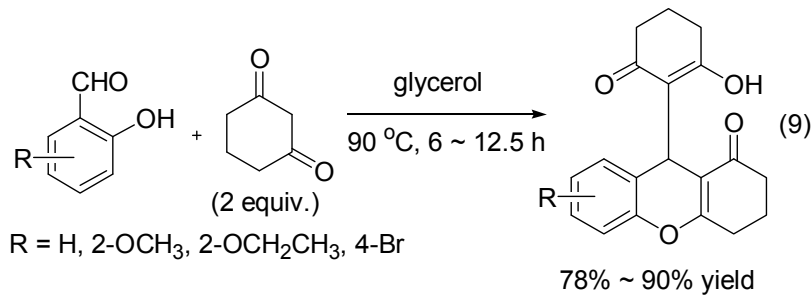

缩硫醛、缩硫酮由于在酸性或碱性条件下的固有稳 定性，常用作有机合成中的羰基保护基 ${ }^{[17]}$. 一般情况 下，缩硫醛化反应通常需要质子酸或路易斯酸作为催化 剂. 然而, 在无催化剂的条件下, 用甘油作溶剂能有效 促进缩硫醛化反应的进行 ${ }^{[18]}$. 在甘油中, 加热到 $90{ }^{\circ} \mathrm{C}$, 醛和酮都能与苯硫酚或 1,2-乙二硫醇发生缩硫醛化反 应, 高产率地得到缩硫醛或缩硫酮(Scheme 3). 反应结 束后, 产物经正已烷萃取即可实现分离, 而甘油可以在 该反应条件下循环使用 4 次以上.
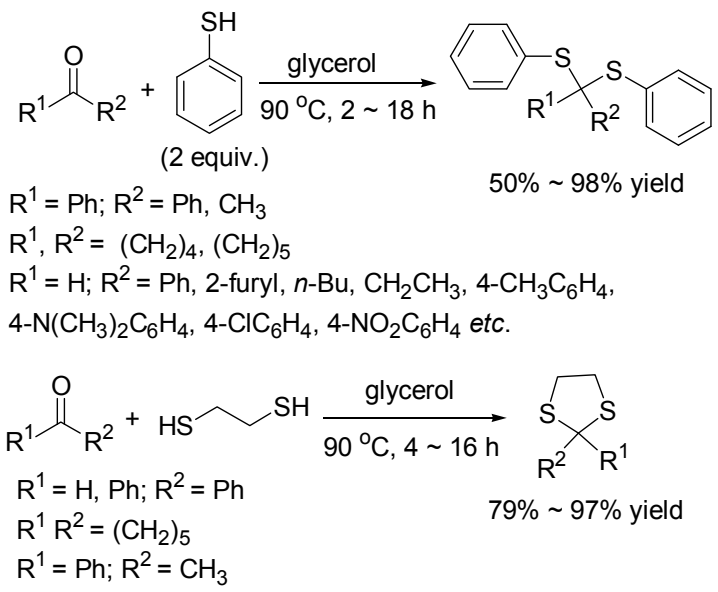

图式 3 甘油中羰基化合物的缩硫醛化反应

Scheme 3 Thioacetalization of carbonyl compounds in glycerol

无溶剂的反应条件下, 定量的 $\operatorname{In}(\mathrm{OTf})_{3}$ 能有效地促 进甘油与非环状缩醛或缩酮发生反缩醛交换反应生成 相应的环状缩醛(Eq. 10 $)^{[19]}$. 该反应的优点在于反应条 件温和(室温), 反应时间快 $(0.5 \mathrm{~h})$, 有较好的转化率 $(\geqslant 85 \%)$ 和较高的化学选择性.

在无催化剂条件下, 在甘油或甘油一水体系中能够 实现苯并咪唑、苯并噻唑、苯并噁唑、喹啞啉类杂环化 合物的合成(Scheme 4) ${ }^{[20 ~ 22]}$. 邻氨基苯硫酚, 邻氨基苯

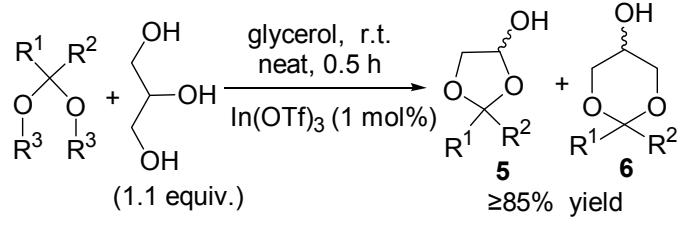

$\mathrm{R}^{1}=\mathrm{Ph} ; \mathrm{R}^{2}=\mathrm{H} ;$ ratio $(\%)(\mathbf{5} / \mathbf{6})=38: 62$

$\mathrm{R}^{1} \mathrm{R}^{2}=\left(\mathrm{CH}_{2}\right)_{5}$; ratio $(\%)(\mathbf{5} / \mathbf{6})>98: 2$

$\mathrm{R}^{1}=\mathrm{R}^{2}=\mathrm{CH}_{3}$; ratio $(\%)(\mathbf{5} / \mathbf{6})>98: 2$

$\mathrm{R}^{3}=\mathrm{Me}$ or $\mathrm{Et}$

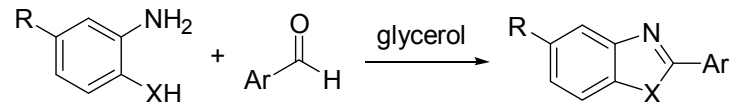

$X=S$, r.t., $0.5 \mathrm{~h}, 80 \% \sim 93 \%$ yield

$\mathrm{R}=\mathrm{Cl}, \mathrm{Ar}=4-\mathrm{CH}_{3} \mathrm{OC}_{6} \mathrm{H}_{4} ; \mathrm{R}=\mathrm{OCH}_{3} ; \mathrm{Ar}=4-\mathrm{NO}_{2} \mathrm{C}_{6} \mathrm{H}_{4}$;

$\mathrm{R}=\mathrm{H} ; \mathrm{Ar}=3-\mathrm{NO}_{2} \mathrm{C}_{6} \mathrm{H}_{4}, 3,4-\left(\mathrm{OCH}_{3}\right)_{3} \mathrm{C}_{6} \mathrm{H}_{2}, 2-\mathrm{HOC}_{6} \mathrm{H}_{4}$;

\section{$\mathrm{X}=\mathrm{NH}, 90^{\circ} \mathrm{C}$, air, $0.7 \sim 6 \mathrm{~h}, 84 \% \sim 94 \%$ yield \\ $\mathrm{R}=\mathrm{H} ; \mathrm{Ar}=\mathrm{Ph}, 2-\mathrm{CH}_{3} \mathrm{OC}_{6} \mathrm{H}_{4}, 4-\mathrm{CH}_{3} \mathrm{C}_{6} \mathrm{H}_{4}, 4-\mathrm{CH}_{3} \mathrm{OC}_{6} \mathrm{H}_{4}$ $4-\mathrm{ClC}_{6} \mathrm{H}_{4}, 4-\mathrm{NO}_{2} \mathrm{C}_{6} \mathrm{H}_{4}$}

$\mathrm{X}=0,90^{\circ} \mathrm{C}, 2 \sim 6 \mathrm{~h}, 70 \% \sim 90 \%$ yield

$\mathrm{R}=\mathrm{H} ; \mathrm{Ar}=\mathrm{Ph}, 4-\mathrm{CH}_{3} \mathrm{C}_{6} \mathrm{H}_{4}, 4-\mathrm{CH}_{3} \mathrm{OC}_{6} \mathrm{H}_{4}, 4-\mathrm{ClC}_{6} \mathrm{H}_{4}$,

4- $\mathrm{BrC}_{6} \mathrm{H}_{4}$, furyl, etc.

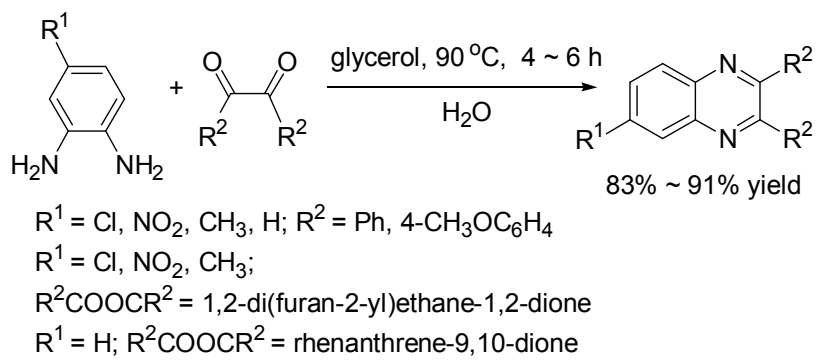

图式 4 苯并咪唑、苯并噻唑、苯并噁唑、喹噁啉的合成 Scheme 4 Synthesis of benzimidazoles, benzothiazoles, benzoxazoles and quinoxalines

酚或邻苯二胺能与醛类化合物反应，以 $70 \%$ ～93\%的产 率生成相应的 2-取代苯并噻唑、苯并噁唑和苯并咪唑衍 生物, 反应对芳香醛、杂环醛和不饱和醛都适用. 在相 同的反应条件下，邻苯二胺与 1,2-二酮也能发生缩合， 以较高的产率生成相应的喹噁啉类衍生物( $83 \% \sim 91 \%)$. 这几个反应都具有适用范围广，反应快速简便等特点. 此外在反应结束后，作为溶剂的甘油均可实现回收循环 使用.

同样，甘油也能对酸催化的脱水二聚反应有明显的 促进作用(Eq. 11) ${ }^{[9]}$ ，而在无溶剂或在水中该反应均不发 生，使用甘油作为溶剂其目标产物产率达 $95 \%$.

\section{5 环合反应}

Colacino 等 ${ }^{[23]}$ 成功地实现了甘油中用钉卡宾配合 物催化的环合反应(RCM). 催化剂(如 $7,8,9)$ 能有效地 催化 $N, N$-二烯丙基磺酰胺发生 RCM 反应(Eq. 12). 该反 

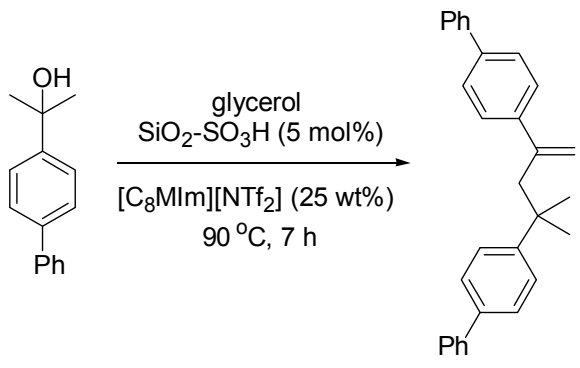

(11)

$95 \%$ yield
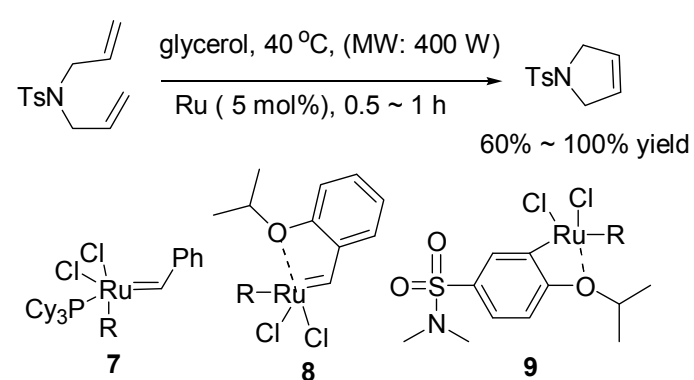

$\mathrm{R}=\mathrm{PCy}_{3}$

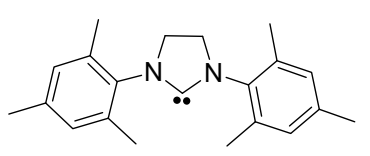

应使用钉作为催化剂, 微波为能量来源, 在 $40{ }^{\circ} \mathrm{C}$ 条件 下, 能快速生成相应的目标产物. 反应结束后, 产物通 过乙醚萃取实现分离, 甘油/钉催化体系可重复使用数 次未见其催化活性降低.

由于呋喃环在天然产物中广泛存在, 因此呋喃环的 合成一直倍受关注, 其中, $(Z)$-烯炔醇的环异构化一直被 认为是合成呋喃环的有效方法 ${ }^{[24,25]}$. 甘油中, 亲水性钯 (II) cis- $\left.\left[\mathrm{PdCl}_{2} \text { (DAPTA }\right)_{2}\right]$ 能够有效地促进(Z)-2-烯-4-炔1-醇衍生物发生环化反应生成相应的呋喃化合物(Eq. $13)^{[26]}$. 虽然该反应在水中进行的反应活性更高, 但以 甘油为溶剂更有利于催化剂的再回收再利用. 以水为溶 剂时, 催化剂的活性随反应的连续进行迅速降低, 最多 能够循环 5 次; 以甘油为溶剂, 该体系即可循环使用 17 次, 其累计转化数(TON)达 8190 .

$$
\begin{aligned}
& \mathrm{R}^{2}=\frac{\text { cis-[PdCl} \left.2(\mathrm{DAPTA})_{2}\right](0.2 \mathrm{~mol} \%)}{\text { glycerol, } 75^{\circ} \mathrm{C}, 0.3 \sim 24 \mathrm{~h}} \mathrm{R}^{1} \\
& \mathrm{R}^{1}=\mathrm{H}, \mathrm{Ph} ; \mathrm{R}^{2}=\mathrm{H}, \mathrm{Ph} \\
& \mathrm{R}^{1}=n-\mathrm{Bu}, \mathrm{CH}_{2} \mathrm{C}\left(\mathrm{CH}_{3}\right)=\mathrm{CH}_{2} ; \mathrm{R}^{2}=\mathrm{H}
\end{aligned}
$$

\section{3 取代反应}

Wolfson 等 ${ }^{[27]}$ 成功实现了苄基氯与硫氰化钾的亲核 取代反应(Eq. 14). 在 $80{ }^{\circ} \mathrm{C}$ 条件下, 以甘油为溶剂在 0.5 $\mathrm{h}$ 内, 硫氰酸苄酯的产率高达 $95 \%$.

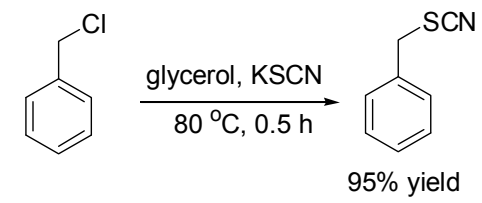

使用甘油作溶剂, 次磷酸 $\left(\mathrm{H}_{3} \mathrm{PO}_{2}\right)$ 作还原剂能有效 地促进 2-氯吡啶与有机二硒醚发生反应, 生成相应的 2有机基硒吡啶(Eq. 15) ${ }^{[28]}$. 该反应在 $\mathrm{N}_{2}$ 氛围, $90{ }^{\circ} \mathrm{C}$ 条件 下反应 $1.5 \sim 5.5 \mathrm{~h}$ 就能得到较高的产率( $70 \% \sim 97 \%$ ). 值 得注意的是, 反应完成后, 产物用 $V($ 己烷 $) / V($ 乙酸乙 酯 $)=95 / 5$ 萃取, 甘油 $/ \mathrm{H}_{3} \mathrm{PO}_{2}$ 体系在真空下干燥后可直 接循环使用 5 次以上, 而且反应无需增加更多的还原剂.

$$
\begin{aligned}
& \mathrm{R}^{1}=\mathrm{Se}_{-} \mathrm{R}^{1}+ \\
& \mathrm{R}^{1}=\mathrm{Ph} ; \mathrm{R}^{2}=\mathrm{H} ; \mathrm{R}^{3}=\mathrm{NH}_{2}, \mathrm{Cl}, \mathrm{H} \\
& \mathrm{R}^{1}=\mathrm{Ph} ; \mathrm{R}^{2}=\mathrm{Cl}_{\mathrm{R}} \mathrm{R}^{3}=\mathrm{H} \\
& \mathrm{R}^{1}=\mathrm{Ph}, 4-\mathrm{CH}_{3} \mathrm{C}_{6} \mathrm{H}_{4}, 2-\mathrm{CH}_{3} \mathrm{C}_{6} \mathrm{H}_{4}, 4-\mathrm{OCH}_{3} \mathrm{C}_{6} \mathrm{H}_{4}, \text { etc.; } \mathrm{R}^{2}=\mathrm{R}^{3}=\mathrm{H}
\end{aligned}
$$

\section{4 氧化反应}

以甘油为溶剂, $\mathrm{Na}_{2} \mathrm{CO}_{3}$ 作碱, $120{ }^{\circ} \mathrm{C}$ 微波辐射 15 $\min$ 后硫醇发生脱氢氧化，以 $81 \% \sim 93 \%$ 的产率得到相 应的二硫化物(Eq. 16 ${ }^{[29]}$. 在该条件下, 芳基硫醇被氧 化成相应的二芳基二硫化物. 值得注意的是，该反应快 速，简便，反应进行完后，用己烷/乙酸乙酯 $(95: 5$, 体 积比)萃取产物, 甘油在真空干燥后可直接循环使用.

$$
\begin{aligned}
& \text { glycerol }(81 \% \sim 93 \% \text { yield })
\end{aligned}
$$

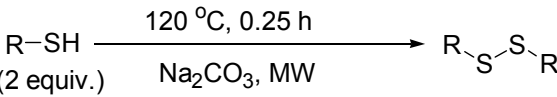

$$
\begin{aligned}
& \mathrm{R}=\mathrm{C}_{6} \mathrm{H}_{5}, \mathrm{CH}_{2} \mathrm{CH}_{3},\left(\mathrm{CH}_{2}\right)_{7} \mathrm{CH}_{3}, \mathrm{C}\left(\mathrm{CH}_{3}\right)_{3} \text {, } \\
& \text { 4- } \mathrm{NH}_{2} \mathrm{C}_{6} \mathrm{H}_{4}, 4-\mathrm{ClC}_{6} \mathrm{H}_{4}, 4-\mathrm{BrC}_{6} \mathrm{H}_{4} \text {, 2-furyl, etc. }
\end{aligned}
$$

\section{5 还原反应}

以甘油为溶剂, 可以实现 $\mathrm{C}=\mathrm{C}$ 键的还原 ${ }^{[30]}$. 此外, Wolfson 等 ${ }^{[27]}$ 以甘油为溶剂, 以计量的硼氢化钠为还原 剂, 成功地实现了苯甲醛的还原(Eq. 17). 进一步研究表 明，在该条件下，其他的羰基化合物如酮、 $\beta$-酩酯等也 可以有选择性地被还原 ${ }^{[31]}$. 该反应具有时间短 $(15 \mathrm{~min})$, 产率高的特点(高达 100\%), 且还原剂的用量可大幅降 低, 0.25 equiv. 的硼氢化钠就可以使乙酰乙酸乙酯完全 转化为 3 -着基丁酸酯.

甘油的沸点高达 $290{ }^{\circ} \mathrm{C}$, 可作为高沸点溶剂在 Wolff-Kishner-黄鸣龙反应中使用 ${ }^{[31]}$. 采用微波技术, 在 


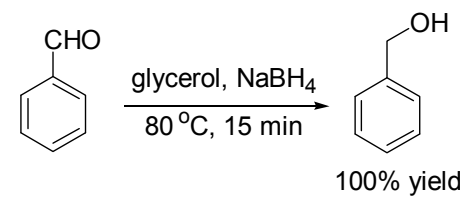

$90{ }^{\circ} \mathrm{C}$ 条件下，5 min 即可完成苯甲醛到甲苯的转化 (Scheme 5).

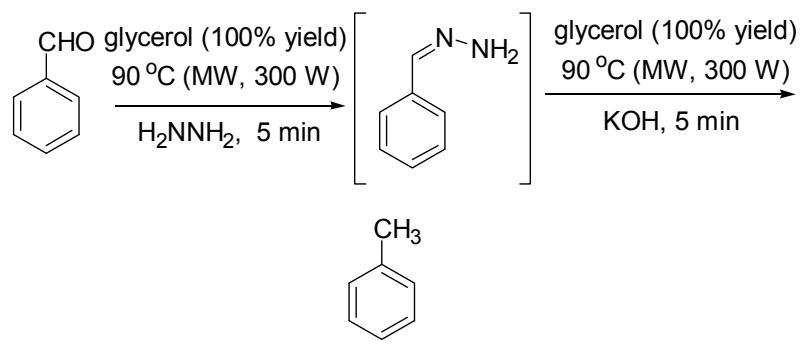

图式 5 苯甲醛的 Wolff-Kishner 还原反应

Scheme 5 Wolff-Kishner reduction of benzaldehyde

甘油作为溶剂也可以在氢化反应中得以应用 ${ }^{[32]}$. Wolfson 等 ${ }^{[27]}$ 在 $80{ }^{\circ} \mathrm{C}, 1.0 \mathrm{MPa} \mathrm{H}$ 条件下, 成功实现了 $\left[\mathrm{RhCl}(\mathrm{TPPTS})_{3}\right]$ 或 $\mathrm{Pd} / \mathrm{C}$ 催化苯乙烯转化为乙基苯. 同 时, 他们实现了甘油中乙酰乙酸乙酯的氢化作用 ${ }^{[31]}$. 尽 管该氢化作用产率极低 $(7 \%)$, 但其对映体过量值(ee 值) 高达 99\%, 同时作为甘油介质中不对称的金属催化的首 例 ${ }^{[33]}$, 为后续工作的开展提供了极其重要的参照.

在过去的几十年中, 金属催化转移氢化反应 $(\mathrm{TH})$ 是 醛、酮、胺和烯烃等有机物还原的有效方法之一 ${ }^{[34]}$. 在 转移氢化的过程中, 甘油除作为反应溶剂外, 还能作氢 的供体, 反应结束后能得到重要的化工原料二羟基丙酮 (DHA) (Eq. 18) ${ }^{[35 \sim 37]}$.

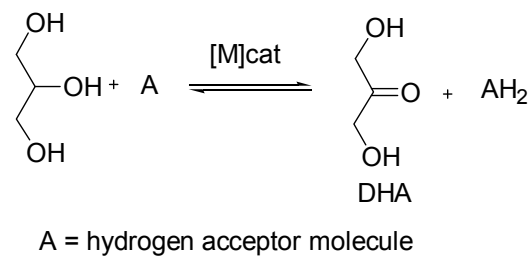

以甘油为溶剂, $100{ }^{\circ} \mathrm{C}$ 条件下, [Ir] 为催化剂, $\mathrm{K}_{2} \mathrm{CO}_{3}$ 为碱, 成功地实现苯乙酮的 $\mathrm{TH}$ 反应, 以 $39 \%$ 的收率得 到相应的苯基乙醇(Eq. 19) ${ }^{[38]}$. 但是研究者发现可能是 由于碱性或者高温的影响, 该条件下 DHA 会发生分解. 为了尽量减少 DHA 的分解, Crotti 等 ${ }^{[39]}$ 探索了铱(I)配合 物(图 3)对苯乙酮的还原能力. 虽然该配合物是异丙醇 中 $\mathrm{TH}$ 反应的高效催化剂 ${ }^{[40]}$, 但在甘油中对苯乙酮几乎 没有还原能力 $($ 产率 $<8 \%=$. 庆幸的是，该配合物对苯 甲醛的还原具有良好的促进作用，能以 $18 \% \sim 46 \%$ 的收 率得到相应的 $\mathrm{TH}$ 产物 ${ }^{[39]}$.

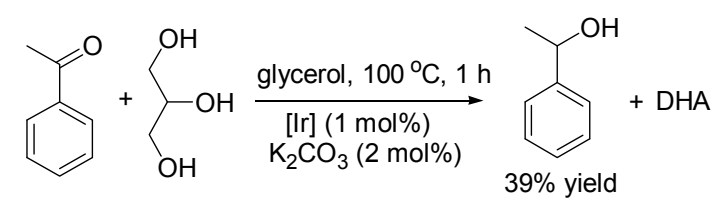

$[\operatorname{lr}]=\left[\operatorname{lr}(1,5\right.$-hexadiene $)\left(\mathrm{Me}_{2}\right.$ phen $\left.)\right][\mathrm{Cl}]$

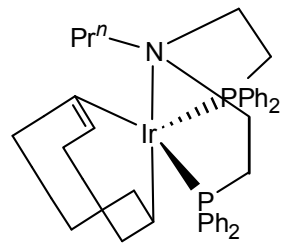

图 3 铱(I)配合物的结构

Figure 3 Structure of the iridium(I) complex

Crotti 等 ${ }^{[41]}$ 将钌二聚体 $\left[\left\{\operatorname{RuCl}(\mu-\mathrm{Cl})\left(\eta^{6}-p\right.\right.\right.$-cymene) $\}_{2}$ ]和 $\mathrm{KOH}$ ( $1 \mathrm{~mol} \%$ 的 Ru, $12 \mathrm{~mol} \%$ 的碱)组成的催 化系统应用到甘油中苯甲醛的转移氢化反应. 不论是采 用微波技术 $(900 \mathrm{~W})$, 还是在常规加热 $\left(60{ }^{\circ} \mathrm{C}\right)$ 条件下， 以 $33 \% \sim 45 \%$ 的产率得到茮醇，只有 $\mathrm{Ru}$ 催化剂的量增 加到 $25 \mathrm{~mol} \%$ 时才可能使苯甲醛发生完全转换(Eq. 20).

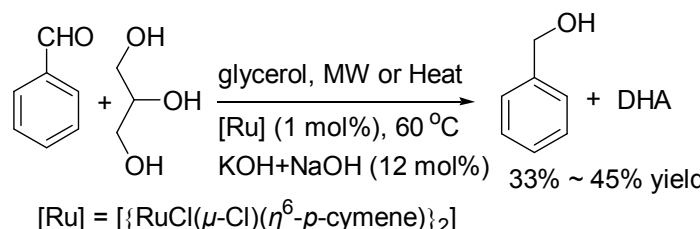

甘油中, 烯烃的转移氢化反应比羰基化合物的更容 易, 以 $\mathrm{Pd} / \mathrm{C}(0.2 \sim 0.3 \mathrm{~mol} \% \mathrm{Pd})$ 为催化剂, 在 $70{ }^{\circ} \mathrm{C}$ 条件 下，环已烯或苯乙烯 5 9 h 内能定量加氢生成相应的烷 烃(Eq. 21) $)^{[41]}$. 但是体积大的烯烃二苯乙烯或 1,1-二苯 基乙烯只能部分还原 $(7 \% \sim 35 \%)^{[42]}$.

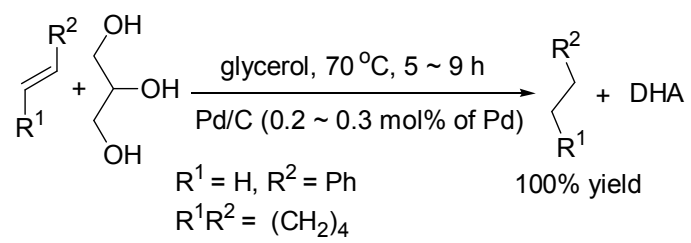

甘油作为溶剂和反应试剂的另一个例子是甘油与 羰基化合物的还原醚化反应(Eq. 22) ${ }^{[43]}$. 在 $\mathrm{H}_{2}$ 条件下, 以 $\mathrm{Pd} / \mathrm{C}$ 和樟脑磺酸为催化剂, 醛或酮和甘油选择性地 发生 1-O-烷基化或 2- $O$-烷基化生成相应的醚类化合物. 甘油三个羟基的 $\mathrm{p} K_{\mathrm{a}}$ 值虽然很相近，可能是由于空间位 阻的关系，导致 1- $O$-烷基化的产物是反应的优势产物.

甘油作为溶剂和氢供体, Raney $\mathrm{Ni} / \mathrm{NaOH}$ 体系可以 高效地还原硝基苯(Eq. 23) ${ }^{[44]}$. 在没有 Raney $\mathrm{Ni}$ 存在的 


$$
\begin{aligned}
& \text { glycerol, } \mathrm{H}_{2}(1.0 \mathrm{MPa}) \\
& 140^{\circ} \mathrm{C}, 24 \mathrm{~h}
\end{aligned}
$$

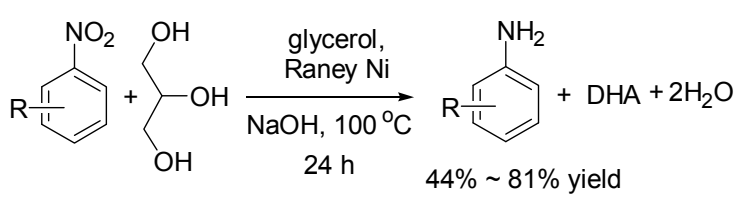

条件下, 硝基苯不会发生还原. 在体系中加入适量的 Raney $\mathrm{Ni}$ 和 $\mathrm{NaOH}$, 于 $100{ }^{\circ} \mathrm{C}$ 下反应 $24 \mathrm{~h}$, 硝基苯的转 化率可达 44\%, 若将反应时间延长至 $78 \mathrm{~h}$, 可实现硝基 苯的完全转化. 反应结束后, 容易实现产物的分离和催 化剂的回收再利用. 反应完毕, 分离出产物后, 向甘油 中加入适量的 $\mathrm{NaOH}$ 即可用于下一循环.

以甘油作溶剂和氢供体, 亲水钌(II)配合物作高效 催化剂, 烯丙醇能定量转化为相应饱和醇 Eq. 24) ${ }^{[40]}$. 该 反应在 $\mathrm{N}_{2}$ 氛围下, $100{ }^{\circ} \mathrm{C}$ 反应 $6 \sim 15 \mathrm{~h}$ 能够实现高效转 化, 芳香族烯丙醇类化合物和脂肪族烯丙醇类化合物均 能实现有效转化. 而且, 在工业级的甘油 $(87 \%$ 纯度)条 件下进行同样的反应也几乎能定量的转化为相应饱和 醇, 这使得从经济角度上具有很好的发展前景. 反应结 束后, 经乙醚萃取分离产物, 甘油相和催化体系可以循 环使用四次以上.

$$
\begin{aligned}
& \int_{\substack{\left.\mathrm{RuCl}\left(\eta^{6} \sim \mathrm{C}_{6} \mathrm{H}_{6}\right)(\mathrm{DAPTA})\right] \\
\mathrm{KOH}(40 \%), \mathrm{N}_{2}}}^{\substack{\text { glycerol }(86 \% \sim 97 \% \text { yield }) \\
100{ }^{\circ} \mathrm{C}, 6 \sim 15 \mathrm{~h}}} \\
& \mathrm{R}=2-\mathrm{CH}_{3} \mathrm{OC}_{6} \mathrm{H}_{4}, 4-\mathrm{CH}_{3} \mathrm{OC}_{6} \mathrm{H}_{4}, 4-\mathrm{FC}_{6} \mathrm{H}_{4} \text {, } \\
& \left(\mathrm{CH}_{2}\right)_{4} \mathrm{CH}_{3},\left(\mathrm{CH}_{2}\right)_{5} \mathrm{CH}_{3}
\end{aligned}
$$

Andrade 等 ${ }^{[45]}$ 发现由 $\mathrm{NaHPO}_{4}$ 和 $\mathrm{KH}_{2} \mathrm{PO}_{4}$ 组成 $\mathrm{pH}=$ 7 的缓冲溶液(PBS)与甘油以 $4: 1$ (体积比)混合作为溶 剂, 用生物酶作催化剂, 在 $32{ }^{\circ} \mathrm{C}$ 条件下经 $48 \mathrm{~h}$ 可将 2氯苯乙酮还原为 2-氯苯基乙醇, 其 $e e$ 高达 99\% (Eq. 25). 其他邻、间和对位取代卤代苯乙酮(氟、氯、溴)在还原 时也得到了良好结果. 然而使用其他有机溶剂 (如 DMSO、THF、乙腈、乙醚等)代替甘油, 并没有得到理 想的结果, 这可能是由于以下原因所致: (1)甘油的加入 有助于反应底物的溶解; (2)与别的有机溶剂不同, 甘油 与水能够混溶, 从而形成一个均相体系, 该体系有助于 反应底物与生物酶的接触; (3) 甘油与 PBS 组成的体系可 以稳定蛋白质天然结构, 并防止其变性, 从而保持酶活
性.

$$
\underset{\substack{\text { Aspergillus terreus } \\ 32^{\circ} \mathrm{C}, 48 \mathrm{~h}}}{\mathrm{PBS} / \text { glycerol }(V: V=4: 1)}
$$

以甘油和 2-甲基四氢呋喃的混合物作双相溶剂, 次 磷酸钠作供氢体, 2,2'-二吡啶(2,2'-bipyridine)作配体, Ru 催化剂能有效地将酮类化合物还原为相应的醇 (Eq. $26)^{[46]}$. 该反应不仅产率令人满意(高达 95\%), 而且具有 优良的化学选择性和对映选择性(97\% ee 值). 芳环上带 有的酯基、㲵基、卤素等在该条件下能够得以保持，脂 肪酮也能很好地参与到反应之中.

$$
\begin{aligned}
& \text { glycerol/2-MeTHF } \\
& 80^{\circ} \mathrm{C}, 18 \sim 24 \mathrm{~h}
\end{aligned}
$$

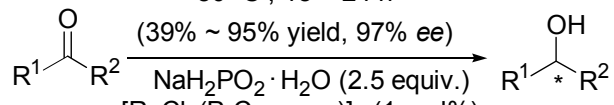

$$
\begin{aligned}
& {\left[\mathrm{RuCl}_{2}(\mathrm{P}-\mathrm{Cymeme})\right]_{2}(1 \mathrm{~mol} \%)} \\
& \mathrm{R}^{1}=\mathrm{R}^{2}=\mathrm{Ph} \\
& \text { bipyr (2.4 mol\%) } \\
& \mathrm{R}^{1}=\mathrm{Ph} ; \mathrm{R}^{2}=\mathrm{CH}_{2} \mathrm{CH}_{2} \mathrm{CH}_{3} \\
& \mathrm{R}^{1}=\mathrm{CH}_{3} ; \mathrm{R}^{2}=\mathrm{Ph}, 4-\mathrm{BrC}_{6} \mathrm{H}_{4}, 4-\mathrm{CNC}_{6} \mathrm{H}_{4} \text { etc. }
\end{aligned}
$$

\section{6 多组分反应}

多组分反应(MCRs) 允许一步操作中含有几种键的 反应, 并且具有操作简单、资源利用率高和高原子经济 性等特点 ${ }^{[47]}$. 由于反应过程中减少了检查、萃取和纯净 化操作的次数, 因而减少了废物的生成, 即实现绿色化; 同时，一锅反应 MCRs 往往缩短反应时间，比多步合成 有更高的总体化学收益. 因此, 新 MCRs 反应与绿色过 程的设计拥有很大的吸引力, 成了有机合成、药物发明 和材料科学领域的研究热点. 在人们为多组分反应寻求 绿色、高效溶剂的时候, 甘油表现出了良好的性质.

$\mathrm{Gu}$ 等 ${ }^{[48]}$ 成功地实现了甘油中 5,5-二甲基-1,3-环己 二酮、多聚甲醛和苯乙烯的多组分反应. 在 $110{ }^{\circ} \mathrm{C}$ 温度 下, 5,5-二甲基-1,3-环已二酮能与多聚甲醛、苯乙烯通过 一个连续 Knoevenagel/hetero-Diels Alder 反应, 以 $68 \%$ 的产率得到最终产物(Eq. 27). 使用甲苯、乙腈、DMF、 DMSO 等作溶剂, 反应也能顺利进行, 但是产率较低.

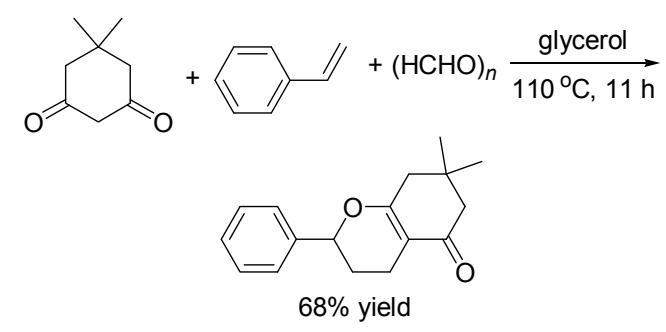

在无催化剂条件下, 以甘油为溶剂, 伯胺、2-芸酚、 
6-甲基-4-差基-2-吡喃酮和 1-甲基-4-差基-2-喹诺酮能与 1,3-环己二酮、甲醛发生多组分反应, 以较高的产率得 到相应的多环化合物(Scheme 6) ${ }^{[48]}$. 在这些 MCRs 中, 使用甲苯、硝基甲烷、DMF 或 DMSO 等典型有机溶剂 也能得到相应的产物, 不过产率相对较低, 这可能是由 于甘油属极性质子性溶剂特性的影响, 使得甘油在 MCRs 中表现出比其他溶剂更明显的效果.

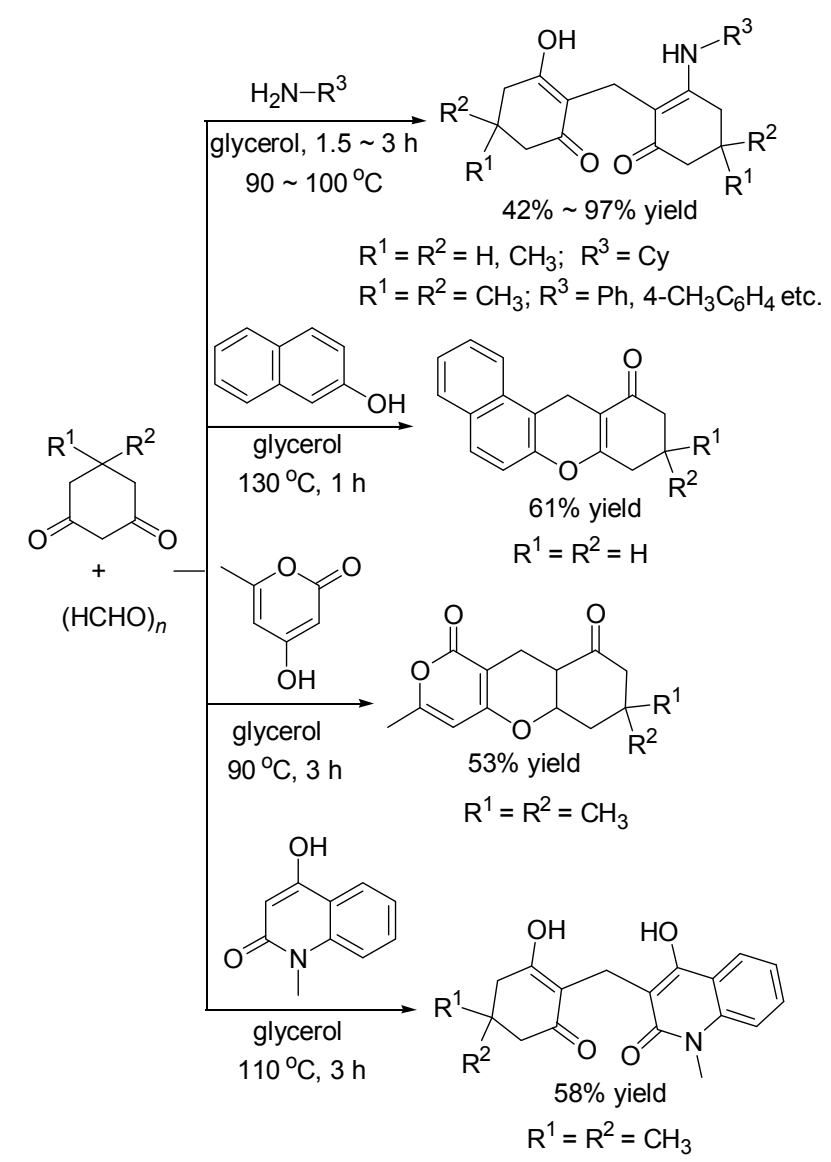

图式 6 甘油中 1,3-环已二酮和甲醛的多组分反应

Scheme 6 Multicomponent reactions of 1,3-cyclohexanediones and formaldehyde in glycerol

甘油作为溶剂被用于苯肼、 $\beta$-酮酸酯、甲醛和苯乙 烯的四组分一锅多步反应之中, 取得了不错的效果 ${ }^{[49]}$. 如 Scheme 7 所示, 第一步反应是在 $110{ }^{\circ} \mathrm{C}$ 条件下, 苯肼 和 $\beta$-酮酸酯在甘油中反应 $4 \mathrm{~h}$, 形成 1,3-二取代-5-吡唑 啉酮; 随后将苯乙烯和多聚甲醛加入到反应混合物中, $110{ }^{\circ} \mathrm{C}$ 条件下反应 $10 \mathrm{~h}$, 通过 Knoevenagel/heteroDiels-Alder 反应生成相应的目标产物.

事实上, 许多反应物, 如 1,3-二羰基化合物、1,3-二 取代-5-吡唑啉酮、2-䒺酚、巴比妥酸等也能与甲醛和苯 乙烯发生多组分反应(MCRs $)^{[50]}$. 同样, 吲哚、芳肼、 $\beta$ 酮酸酯和多聚甲醛也能发生多组分反应生成多取代吲 哚衍生物(Scheme 8) ${ }^{[49]}$. 无独有偶, 在这些反应中甘油
作为溶剂都显示了独特的优势.

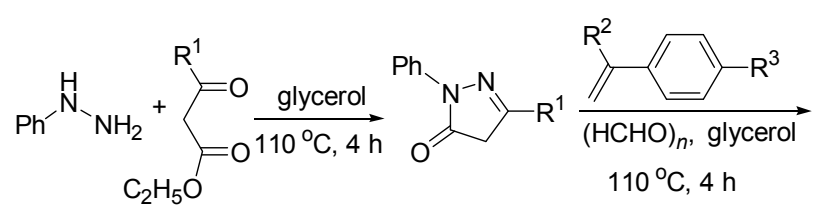

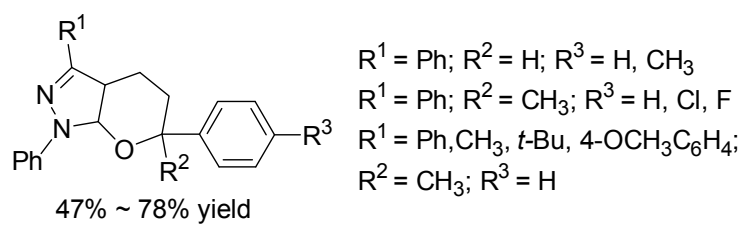

图式 7 甘油中的四组分一锅多步反应

Scheme 7 Four-component one-pot, two-step reaction in glycerol

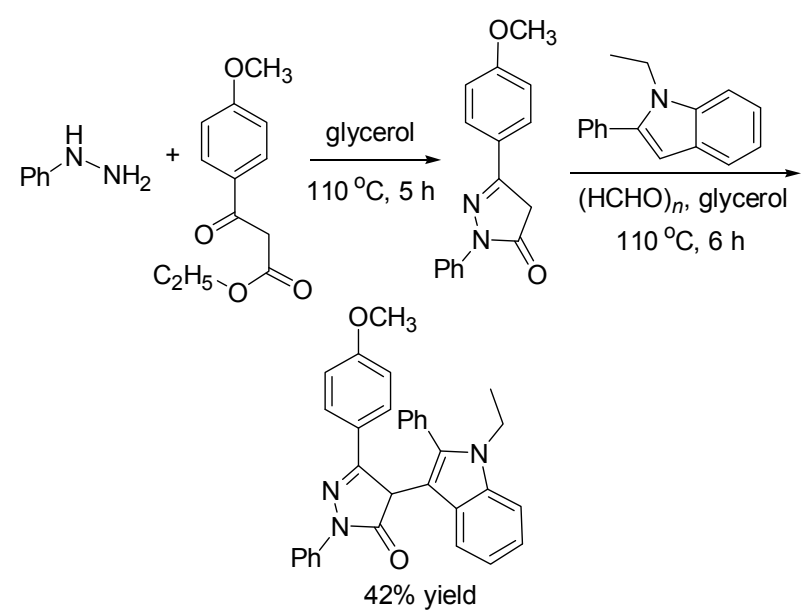

图式 8 多取代吲哚的合成

Scheme 8 Synthesis of the functionalized indole

以芳醛、米氏酸和 2-䒺胺为原料, 在 $110{ }^{\circ} \mathrm{C}$ 条件下, 甘油作为有机反应溶剂和催化剂, 通过三组分一锅反 应，合成一系列的 1-芳基苯并 $[f]$ 喹啉衍生物 (Eq. $28)^{[51,52]}$. 无论是苯甲醛或是杂环醛，邻位、间位还是对 位，均有比较好的产率 $(80 \% \sim 86 \%)$. 此外，该方法具有 反应条件温和、产率较高、后处理简单、环境友好等优 点.

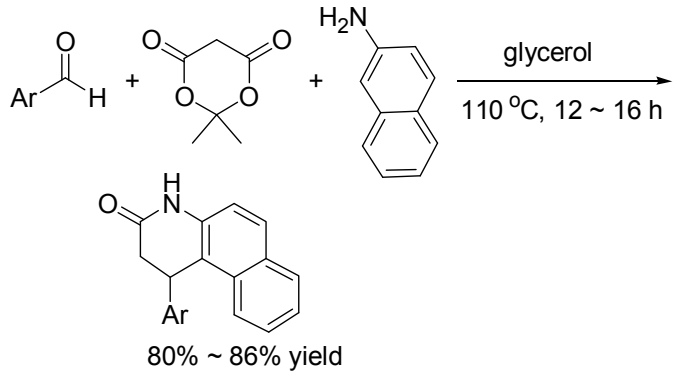

$\mathrm{Ar}=\mathrm{Ph}, 4-\mathrm{FC}_{6} \mathrm{H}_{4}, 4-\mathrm{BrC}_{6} \mathrm{H}_{4}, 4-\mathrm{ClC}_{6} \mathrm{H}_{4}, 4-\mathrm{NO}_{2} \mathrm{C}_{6} \mathrm{H}_{4}$, 3- $\mathrm{NO}_{2} \mathrm{C}_{6} \mathrm{H}_{4}, 4-\mathrm{CH}_{3} \mathrm{OC}_{6} \mathrm{H}_{4}$ etc.

以甘油为溶剂, 聚苯乙烯-PEG 固载磺酸(PS-PEGO $\mathrm{SO}_{3} \mathrm{H}$ ) 为绿色可回收催化剂, 通过多组分反应可合成 
3,4-二氢嘧啶酮衍生物 ${ }^{[53]}$. 在 $100{ }^{\circ} \mathrm{C}$ 条件下, 乙酰乙酸 乙酯、苯甲醛和尿素(或硫艮)通过 Biginelli 反应, $8 \mathrm{~h}$ 内 以 75\% 93\%的产率得到相应的 3,4-二氢嘧啶酮衍生物 (Eq. 29). 反应结束后, PS-PEGOSO ${ }_{3} \mathrm{H}$ 经过过滤和洗涤 后可回收循环使用, 甘油经减压浓缩后也可循环使用.

$$
\text { 年 }
$$

$\mathrm{X}=\mathrm{O} ; \mathrm{Ar}=\mathrm{C}_{6} \mathrm{H}_{5}, 4-\mathrm{CH}_{3} \mathrm{OC}_{6} \mathrm{H}_{4}, 4-\mathrm{NO}_{2} \mathrm{C}_{6} \mathrm{H}_{4}, 2-\mathrm{ClC}_{6} \mathrm{H}_{4}$ $\mathrm{X}=\mathrm{S} ; \mathrm{Ar}=\mathrm{C}_{6} \mathrm{H}_{5}, 4-\mathrm{CH}_{3} \mathrm{OC}_{6} \mathrm{H}_{4}, 3-\mathrm{NO}_{2} \mathrm{C}_{6} \mathrm{H}_{4}, 4-\mathrm{NO}_{2} \mathrm{C}_{6} \mathrm{H}_{4}$ 4- $-\mathrm{ClC}_{6} \mathrm{H}_{4}, 4-\mathrm{CH}_{3} \mathrm{C}_{6} \mathrm{H}_{4}$ etc.

以甘油为溶剂, $\mathrm{KF} / \mathrm{Al}_{2} \mathrm{O}_{3}$ 为催化剂, 在 $\mathrm{N}_{2}$ 保护条件 下，芳醛、芳酮和硫醇通过一锅反应高产率地合成 $\beta$-芳 基- $\beta$-硫酮衍生物(55\% 78\%) (Scheme 9) ${ }^{[54]}$. 反应具有 适用范围广、操作简便等优点. 反应结束后, 将反应混 合物用己烷/乙酸乙酯(95:5, 体积比)萃取, 即可得到 目标产物，甘油可直接用于下一次反应.

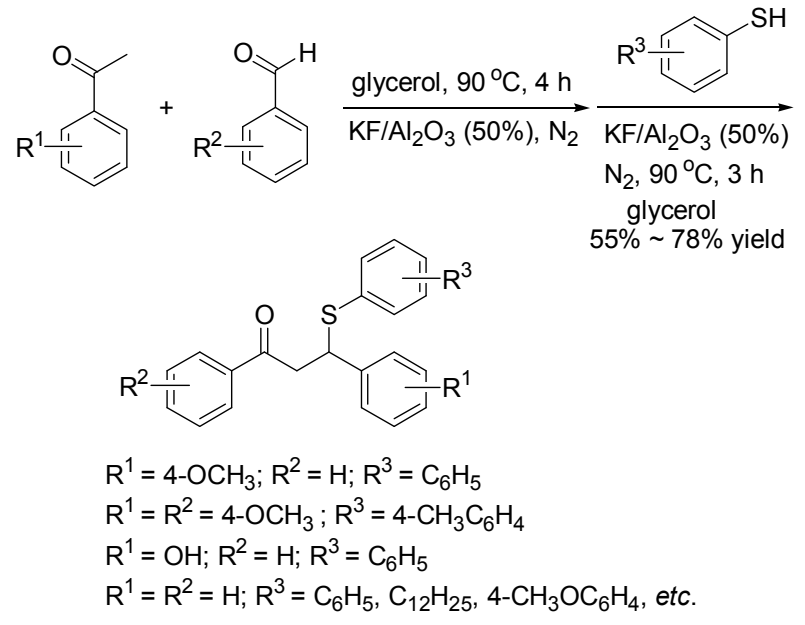

图式 $9 \beta$-芳基- $\beta$-硫酮衍生物的合成

Scheme 9 Synthesis of $\beta$-aryl- $\beta$-sulfanyl ketones

1,4-二氢吡啶由于具有重要的生物活性, 其合成受 到了广泛关注 ${ }^{[55]}$, 最常用的方法之一是 Hantzsch 反应,
即一分子的醛、两分子的 $\beta$-酮酸酯和一分子的铵盐之间 通过三组分四分子的一锅法反应. Nagaiah 等 ${ }^{[56]}$ 选用氯 化铈为催化剂, 甘油为溶剂, 成功实现了乙酰乙酸乙 酯、乙酸铵和醛类化合物的 Hantzsch 反应，以 $80 \%$ 95\%的产率得到相应的 1,4-二氢吡啶(Eq. 30). 该方法适 用范围广，各种富电子的或缺电子的芳香族醛、芳杂醛 或脂族醛都能顺利转化.
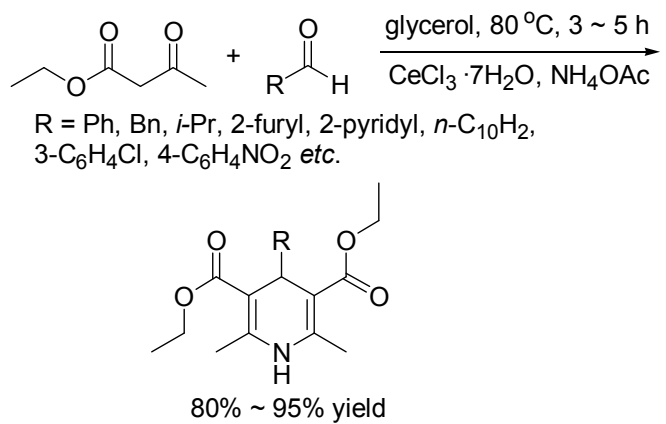

\section{7 偶联反应}

2007 年, Wolfson 等 ${ }^{[28,57]}$ 成功地证明了甘油是 Heck 反应的良好介质(Eq. 31). 他们以碘苯和丙烯酸正丁酯 的反应为研究对象, 考察了几种钯试剂的催化活性. 其 结果表明, 在使用 $\mathrm{PdCl}_{2} 、 \mathrm{Pd}(\mathrm{OAc})_{2}$ 或 $\left[\mathrm{Pd}(\mathrm{OAc})_{2}-\right.$ (TPPTS $)_{2}$ ] [TPPTS: 三(3-磺基苯基)磷三钠盐]作催化剂 时, 效果较好, $80{ }^{\circ} \mathrm{C}$ 加热 $4 \mathrm{~h}$ 后, 目标产物的产率为 $32 \% \sim 100 \%$.

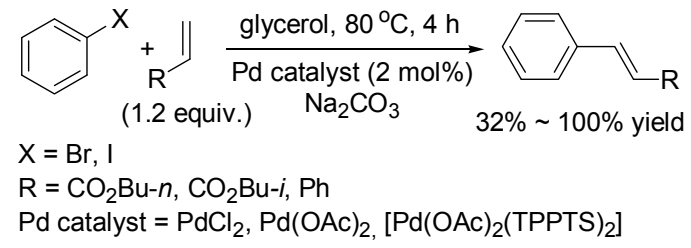

Jérôme 等 ${ }^{[58]}$ 以过量的碘代芳烃与丙烯酸酯为原料, 在甘油中通过两次 Heck 反应实现了丙烯酸酯的二芳基 化. 虽说该条件下有少量自身偶联产物 $(<10 \%)$ 生成， 但没有发现单芳基化产物. 相比水溶液中的反应，二芳 基烯烃在甘油中有良好的收率，可能是因为酯基易发生 部分水解所致 ${ }^{[59]}$. 有趣的是, 在相同条件下, 分步加入 不同的碘代芳烃，可以实现丙烯酸酯的不对称二芳基化 (Scheme 10).

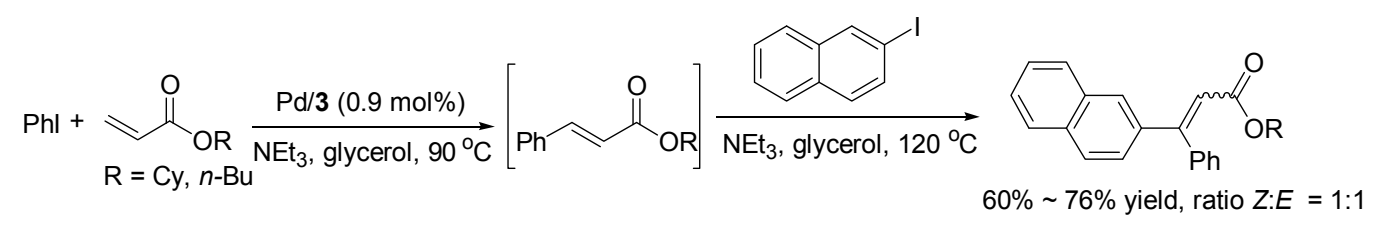

图式 10 丙烯酸酯的不对称芳基化反应

Scheme 10 Unsymmetrical diarylations of acrylates 
以甘油作溶剂, 可实现碘苯与苯基硼酸的 Suzuki 反 应 ${ }^{[49]}$. Azua 等 ${ }^{[60]}$ 以甘油为溶剂, $\mathrm{K}_{2} \mathrm{CO}_{3}$ 作缚酸剂, $\mathrm{N}$-杂环 卡宾钯(Pd-NHC)作催化剂, 在超声(US)条件下实现溴代 芳烃、碘代芳烃与芳基硼酸、芳基硼酸盐的交叉偶联反 应，以较高的产率 $(68 \% \sim 90 \%)$ 得到相应的联芳烃类化 合物(Eq. 32). 反应简洁高效, 所有反应在半小时内即可 完成.
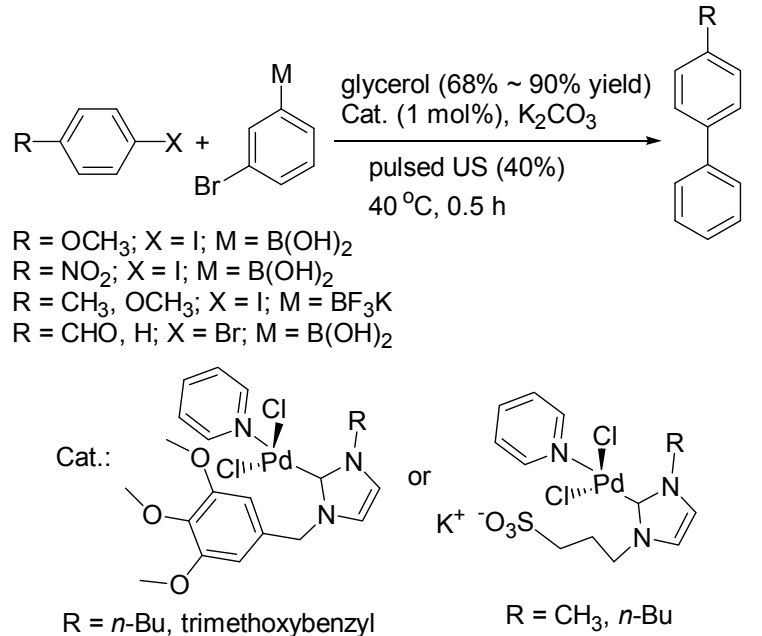

以甘油作溶剂, $\mathrm{CuI}$ 作催化剂, DMSO 作共溶剂, 二 芳基二硒醚能与芳基嗍酸发生交叉偶联反应生成相应 的二芳基硒化物(Eq. 33) ${ }^{[61]}$. 该反应无需惰性气体保护, 在空气氛围中 $110{ }^{\circ} \mathrm{C}$ 条件下反应 $30 \mathrm{~h}$, 以 $73 \% \sim 90 \%$ 产 率得到相应目标产物. 反应结束后, 将反应混合物用水 稀释并用己烷/乙酸乙酯 $(95: 5$, 体积比) 萃取即可实现 产物的分离, 甘油/CuI 的混合物可以直接再用于下一步 反应.

$$
\begin{aligned}
& \text { glycerol }(73 \% \sim 90 \% \text { yield })
\end{aligned}
$$

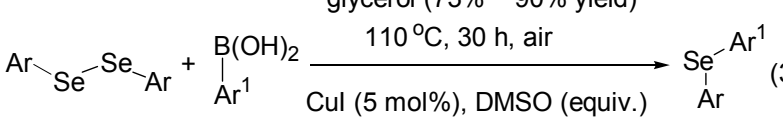

$$
\begin{aligned}
& \mathrm{Ar}=\mathrm{Ph} ; \mathrm{Ar}^{1}=\mathrm{Ph}, 2-\mathrm{CH}_{3} \mathrm{OC}_{6} \mathrm{H}_{4}, 4-\mathrm{CH}_{3} \mathrm{C}_{6} \mathrm{H}_{4}, 2-\mathrm{CH}_{3} \mathrm{C}_{6} \mathrm{H}_{4} \text {, } \\
& \text { 4- }-\mathrm{ClC}_{6} \mathrm{H}_{4}, 2-\mathrm{ClC}_{6} \mathrm{H}_{4}, 4-\mathrm{BrC}_{6} \mathrm{H}_{4}, 2-\mathrm{BrC}_{6} \mathrm{H}_{4}, 3-\mathrm{CF}_{3} \mathrm{C}_{6} \mathrm{H}_{4} \text {; } \\
& \mathrm{Ar}=\mathrm{Ph}, 4-\mathrm{CH}_{3} \mathrm{OC}_{6} \mathrm{H}_{4}, 4-\mathrm{CH}_{3} \mathrm{C}_{6} \mathrm{H}_{4}, 2-\mathrm{CH}_{3} \mathrm{C}_{6} \mathrm{H}_{4}, 4-\mathrm{ClC}_{6} \mathrm{H}_{4} \text {, } \\
& \text { 3- }-\mathrm{CF}_{3} \mathrm{C}_{6} \mathrm{H}_{4}, 2,4,6-\left(\mathrm{CH}_{3}\right)_{3} \mathrm{C}_{6} \mathrm{H}_{2} ; \mathrm{Ar}^{1}=4-\mathrm{CH}_{3} \mathrm{OC}_{6} \mathrm{H}_{4}
\end{aligned}
$$

以甘油作溶剂, $\mathrm{CuI}$ 作催化剂, DMSO 作添加剂, $\mathrm{K}_{2} \mathrm{CO}_{3}$ 作缚酸剂, 可以实现吲哚和芳基卤化物的交叉偶 联反应(Eq. 34) ${ }^{[62]}$. 反应在 $120{ }^{\circ} \mathrm{C}$ 条件下, 反应 $24 \mathrm{~h}$ 能 够得到相应的 $N$-芳基吲哚, 在相同条件下, 吡咯、咪唑、 吡唑衍生物均能与碘苯发生相应的 $N$-芳基化反应. 反应 结束后, 用乙醚萃取即可实现产物的分离, 而甘油/CuI 体系能够重复循环使用四次以上.

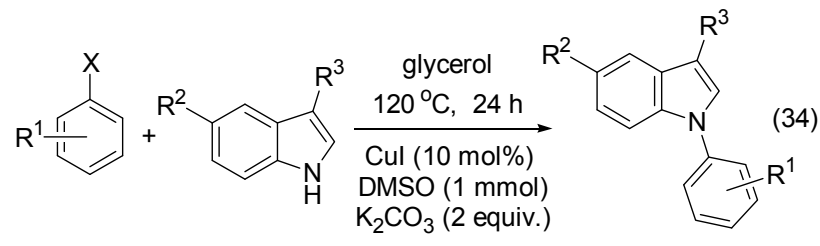

$\mathrm{X}=\mathrm{I}, \mathrm{Br} ; \mathrm{R}^{1}=\mathrm{CH}_{3}, \mathrm{OCH}_{3}, \mathrm{Br}, \mathrm{F}, \mathrm{NO}_{2}$

$\mathrm{R}^{2}=\mathrm{OCH}_{3}, \mathrm{Br}, \mathrm{NO}_{2} ; \mathrm{R}^{3}=\mathrm{CH}_{3}$

$65 \% \sim 92 \%$ yield

\section{8 结束语}

甘油作为绿色溶剂在有机合成中得以应用, 不仅为 利用生物柴油产业产生的副产物提供了新途径, 而且也 为溶剂的革新提供了许多新的机遇. 它具有价廉、无毒、 不易燃和可生物降解性等特点, 使其有希望成为绿色合 成产业的理想溶剂. 然而, 它的高粘性可能会导致反应 物在介质中的扩散作用较差; 当用甘油作为一个特定反 应的溶剂时，它的三个高活性羟基可能优先发生反应; 如何从甘油中提取极性大的反应产物; 以及甘油促进作 用的原理都是进行大规模的实际应用之前需要解决的 问题. 综上所述，即使有众多成功事例，甘油作为绿色 溶剂替代传统有机溶剂, 大规模地应用于有机合成化学 在未来还需要作出很多努力.

\section{References}

[1] Kerton, F. M. In Alternative Solvents for Green Chemistry, RSC Green Chemistry Series, Ed.: Clark, J., RSC Publishing, Cambridge, 2009

[2] Capello, C.; Fisher, U.; Kungerbühler, K. Green Chem. 2007, 9, 927.

[3] (a) DeSimone, J. M. Science 2002, 297, 799.

(b) Nelson, W. M. Green Solvents for Chemistry: Perspectives and Practice, Oxford University Press, New York, 2003.

(c) Clark, J. H.; Taverner, S. J. Org. Process Res. Dev. 2007, 11, 149 .

(d) Kerton, F. M. Alternative Solvents for Green Chemistry, RSC Publishing, Cambridge, 2009.

[4] Palkovits, R.; Tajvidi, K.; Ruppert, A. M. Chem. Commun. 2011, $47,576$.

[5] (a) Kemp, W. H. Biodiesel: Basics and Beyond, Azlext Press, Ontario, 2006

(b) Demirbas, A. Biodiesel: A Realistic Fuel Alternative for Diesel Engines, Springer-Verlag, London, 2008.

(c) Pahl, G. Biodiesel: Growing A New Energy Economy, White River Junction, Chelsea Green Publishers, VT, 2005.

(d) Knothe, G.; Krahl, J.; Gerpen, J. V. The Biodiesel Handbook, AOCS Publishing, Urbana, 2010.

(e) Hoogendoorn, A.; van Kasteren, H. Transportation Biofuels Novel Pathways for the Production of Ethanol, Biogas and Biodiesel, RSC Publishing, Cambridge, 2010.

[6] Pagliaro, M.; Rossi, M. The Future of Glycerol: New Usages for a Versatile Raw Material, RSC Publishing, Cambridge, 2008.

[7] Wolfson, A.; Dlugy, C.; Tavor, D. Tetrahedron: Asymmetry 2006, 17, 2043.

[8] (a) Gu, Y.; Jérôme, F. Green Chem. 2010, 12, 1127. 
(b) Cintas, P.; Tagliapietra, S.; Calcio Gaudino, E.; Palmisanoc, G.; Cravotto, G. Green Chem. 2014, 16, 1056.

(c) García, J. I.; García-Marín, H.; Pires, E. Green Chem. 2014, 16, 1007.

[9] Gu, Y.; Barrault, J.; Jérôme, F. Adv. Synth. Catal. 2008, 350, 2007.

[10] Lenardão, E. J.; Trecha, D. O.; Ferreira, P. C.; Jacob, R. G.; Perin, G. J. Braz. Chem. Soc. 2009, $20,93$.

[11] Wolfson, A.; Litvak, G.; Dlugy, C.; Shotland, Y.; Tavor, D. Ind. Crops Prod. 2009, 30, 78.

[12] Zhang,-M. J. Nantong Vocational College 2009, 23(4), 95 (in Chinese).

(张民，南通职业大学学报, 2009, 23(4), 12.)

[13] Karam, A.; Villandier, N.; Delample, M.; Koerkamp, C. K.; Douliez, J. P.; Granet, R.; Krausz, P.; Barrault, J.; Jérôme, F. Chem.-Eur. J. 2008, 14, 10196.

[14] Balieu, S.; El Zein, A.; De Sousa, R.; Jérôme, F.; Tatibouët, A.; Gatard, S.; Pouilloux, Y.; Barrault, J.; Rollin, P.; Bouquillon, S. Adv. Synth. Catal. 2010, 352, 1826.

[15] He, F.; Li, P.; Gu, Y.; Li, G. Green Chem. 2009, 11, 1767.

[16] Silveira, C. C.; Mendes, S. R.; Lībero, F. M.; Lenardão, E. J.; Perin, G. Tetrahedron Lett. 2009, 50, 6060.

[17] (a) Kocienski, P. J. Protecting Groups, 3rd ed., Thieme Verlag, Stuttgart, 2003.

(b) Greene, T. W.; Wuts, P. G. M. Protective Groups in Organic Synthesis, 3rd ed., Wiley-Interscience, New York, 1999.

[18] Perin, G.; Mello, L. G.; Radatz, C. S.; Savegnago, L.; Alves, D.; Jacob, R. G.; Lenardão, E. J. Tetrahedron Lett. 2010, 51, 4354.

[19] Smith, B. M.; Kubczyk, T. M.; Graham, A. E. RSC Adv. 2012, 7, 2702.

[20] (a) Bachhav, H. M.; Bhagat, H. M.; Telvekar, V. N. Tetrahedron Lett. 2011, 52, 5697.

(b) Sadek, K. U.; Mekheimer, R. A.; Hameed, A. M. A.; Elnahas, F.; Elnagdi, M. H. Molecules 2012, 17, 6011.

[21] (a) Zhang, X.-Z.; Zhou, W.-J.; Yang, M.; Wang, J.-X. J. Chem. Res. 2012, 489.

(b) Zhou, W.-J.; Zhang, X.-Z.; Sun, X.-B.; Wang, J.-X.; Bai, L. Russ. Chem. Bull. 2013, 62, 1244.

[22] Radatz, C. S.; Silva, R. B.; Perin, G.; Lenardão, E. J.; Jacob, R. G.; Alves, D. Tetrahedron Lett. 2011, 52, 4132.

[23] Bakhrou, N.; Lamaty, F.; Martinez, J.; Colacino, E. Tetrahedron Lett. 2010, 51, 3935.

[24] (a) Kirsch, S. F. Org. Biomol. Chem. 2006, 4, 2076.

(b) D'Souza, D. M.; Müller, T. J. J. Chem. Soc. Rev. 2007, 36, 1095.

(c) Balme, G.; Bouyssi, D.; Monteiro, N. Heterocycles 2007, 73, 87.

(d) Cadierno, V.; Crochet, P. Curr. Org. Synth. 2008, 5, 343.

(e) Perin, G.; Mello, L. G.; Radatz, C. S. Tetrahedron Lett. 2010, 51, 4354.

[25] (a) Özdemir, I.; Yiqit, B.; Ülkü, D.; Tahir, M. N.; Arıcı, C. J. Organomet. Chem. 2001, 633, 27.

(b) Diáz-Álvarez, A. E.; Crochet, P.; Zablocka, M.; Duhayon, C.; Cadierno, V.; Gimeno, J.; Majoral, J. P. Adv. Synth. Catal. 2006, 348, 1671.

(c) Garcıá-Garrido, S. E.; Francos, J.; Cadierno, V.; Basset, J. M.; Polshettiwar, V. ChemSusChem 2011, 4, 104.

[26] Francos, J.; Cadierno, V. Green Chem. 2010, 12, 1552.

[27] Wolfson, A.; Dlugy, C.; Shotland, Y. Environ. Chem. Lett. 2007, 5, 67.

[28] Thurow, S.; Webber, R.; Perin, G.; Lenardão, E. J.; Alves, D. Tetrahedron Lett. 2013, 54, 3215

[29] Cabrera, D. M. L.; Líbero, F. M.; Alves, D.; Perin, G.; Lenardão, E. J.; Jacob, R. G. Green Chem. Lett. Rev. 2012, 5, 329.

[30] Aldea, L.; Fraile, J. M.; Garcıá-Marỉn, H.; Garcıá, J. I.; Herrerıás,
C. I.; Mayoral, J. A.; Pérez, I. Green Chem. 2010, 12, 435.

[31] Wolfson, A.; Dlugy, C. Org. Commun. 2009, 2, 34.

[32] (a)Tarama, K.; Funabiki, T. Bull. Chem. Soc. Jpn. 1968, 41, 1744. (b) Funabiki, T.; Tarama, K. Bull. Chem. Soc. Jpn. 1971, 44, 945.

[33] Spinaceánd, E. V.; Vaz, J. M. Catal. Commun. 2003, 4, 91.

[34] (a) Samec, J. S. M.; Bäckvall, J. E.; Andersson, P. G.; Brandt, P. Chem. Soc. Rev. 2006, 35, 237.

(b) Ikariya, T.; Blacker, A. J. Acc. Chem. Res. 2007, 40,1300.

[35] Díaz-Álvarez, A. E.; Cadierno, V. Appl. Sci. 2013, 3, 55.

[36] Elofson, R. M.; Rossow, A. G.; Robinson, C. C. J. Am. Chem. Soc. 1949, 71, 3622.

[37] Miltenberger, K. In Ullman's Encyclopedia Industrial Chemistry, Vol. 13, Eds.: Elvers, B.; Hawkins, S.; Ravenscroft, M.; Rounsaville, J. F.; Schulz, G., Wiley-VCH, Weinheim, 1989, p. 507.

[38] Farnetti, E.; Kašpar, J.; Crotti, C. Green Chem. 2009, 11, 704.

[39] Crotti, C.; Kašpar, J.; Farnetti, E. Green Chem. 2010, 12, 1295.

[40] (a) Bianchini, C.; Farnetti, E.; Graziani, M.; Nardin, G.; Vacca, A.; Zanobini, F. J. Am. Chem. Soc. 1990, 112, 9190.

(b) Farnetti, E.; Gulati, N. V.; Graziani, M. Gazz. Chim. Ital. 1993, 123, 165.

[41] (a) Wolfson, A.; Dlugy, C.; Shotland, Y.; Tavor, D. Tetrahedron Lett. 2009, 50, 5951.

(b) Tavor, D.; Sheviev, O.; Dlugy, C.; Wolfson, A. Can. J. Chem. 2010, $88,305$.

[42] Tavor, D.; Popov, S.; Dlugy, C.; Wolfson, A. Org. Commun. 2010, 3,70 .

[43] (a) Shi, Y.; Dayoub, W.; Favre-Réguillon, A.; Chen, G. R.; Lemaire, M. Tetrahedron Lett. 2009, 50, 6891.

(b)Shi, Y.; Daoub, W.; Chen, G. C.; Lemaire, M. Green Chem. 2010, 12,2189

[44] Tavor, D.; Gefen, I.; Dlugy, C.; Wolfson, A. Synth. Commun. 2011, 41, 3409 .

[45] Andrade, L. H.; Piovan, L.; Pasquini, M. D. Tetrahedron: Asymmetry 2009, 20, 1521.

[46] Guyon, C.; Métay, E.; Duguet, N.; Lemaire, M. Eur. J. Org. Chem. 2013, 5439.

[47] Zhu, J.; Bienaymé, H. Multicomponent Reactions, Wiley-VCH, Weinheim, 2005

[48] Li, M.; Chen, C.; He, F.; Gu, Y. Adv. Synth. Catal. 2010, 352, 519.

[49] Tan, J. N.; Li, M.; Gu, Y. Green Chem. 2010, 12, 908.

[50] (a) Gu, Y.; De, S. R.; Frapper, G.; Bachmann, C.; Barrault, J.; Jéřome, F. Green Chem. 2009, 11, 1968.

(b) Gu, Y.; Barrault, J.; Jéřome, F. Adv. Synth. Catal. 2009, 351, 3269 .

[51] Wang, X.-M. J. Lanzhou Univ. Technol. 2012, 38, 58 (in Chinese). (王雪梅, 兰州理工大学学报, 2012, 38, 58.)

[52] (a) Zhang, M.-M.;Wang, X.-S.; Li, Q. J. Org. Chem. 2008, 28, 881 (in Chinese).

(张梅梅, 王香善, 李庆, 有机化学, 2008, 28, 881.)

(b) Wang, X.-C.; Wang, X.-M.; Quan, Z.-J. J. Northwest Normal Univ.: Nat. Sci. 2011, 47, 60 (in Chinese).

(王喜存, 王雪梅, 权正军, 西北师范大学学报: 自然科学版, 2011, 47, 60.)

[53] Quan, Z. J.; Ren, R. G.; Da, Y. X.; Zhang, Z.; Wang, X. C. Synth. Commun. 2011, 41, 3106.

[54] Perin, G.; Mesquita, K.; Calheiro, T. P.; Silva, M. S.; Lenardão, E. J.; Alves, D.; Jacob, R. G. Synth. Commun. 2014, 44, 49.

[55] Debache, A.; Ghalem, W.; Boulcina, R.; Belfaitah, A.; Rhouati, S.; Carboni, B. Tetrahedron Lett. 2009, 50, 5248.

[56] Narsaiah, A. V.; Nagaiah, B. Asian J. Chem. 2010, 22, 8099.

[57] Wolfson, A.; Dlugy, C. Chem. Pap. 2007, 61, 228.

[58] Delample, M.; Villandier, N.; Douliez, J. P.; Camy, S.; Condoret, J. 
S.; Pouilloux, Y.; Barrault, J.; Jérôme, F. Green Chem. 2010, 12, 804.

[59] Botella, L.; Najera, C. J. Org. Chem. 2005, 70, 4360.

[60] Azua, A.; Mata, J. A.; Heymes, P.; Peris, E.; Lamaty, F.; Martinez, J.; Colacino, E. Adv. Synth. Catal. 2013, 355, 1107.
[61] Ricordi, V. G.; Freitas, C. S.; Perin, G.; Lenardão, E. J.; Jacob,R. G.; Savegnago, L.; Alves, D. Green Chem. 2012, 14, 1030.

[62] Yadav, D. K. T.; Rajak, S. S.; Bhanage, B. M. Tetrahedron Lett. 2014, 55, 931. 